\title{
Calculation of the Streaming Potential Near a Rotating Disk with Rotational Elliptic Coordinates
}

\author{
F.S. Lameiras ${ }^{*}$ and E.H.M. Nunes \\ Centro de Desenvolvimento da Tecnologia Nuclear, Comissão Nacional de Energia Nuclear, \\ Av. Antônio Carlos, 6.627, 31.270-901 Belo Horizonte, Minas Gerais, Brazil \\ Received $23^{\text {rd }}$ January 2008; accepted $10^{\text {th }}$ March 2008
}

\begin{abstract}
The calculation with rotational elliptic coordinates of the streaming potential in the vicinity of a disk-shaped sample rotating in an electrolytic solution is presented. The measurement of this streaming potential is used to determine the zeta potential of planar surfaces. Rotational elliptic coordinates are favored in relation to integral transform methods because only simple mathematical methods are employed to explain the theory of this technique.
\end{abstract}

Keywords: zeta potential, planar surfaces, rotating disk, streaming potential, rotational elliptic coordinates.

\section{Introduction}

A new method and a new apparatus to measure the zeta potential of a planar surface, $\zeta$, based on the measurement of the electric potential in the vicinity of a rotating disk were described in three previous papers [1,2,3]. A sample in the shape of a disk is fixed to a rotating cylindrical support immersed in a fluid with electric charges. The rotation of the disk generates a radial movement of electric charges in the diffuse layer adjacent to the sample's face, resulting in an electric potential distributed in the space near the sample. This electric potential can be measured and is proportional to the zeta potential. The advantage of this new method is the experimental simplicity, which allows the construction of a reliable equipment.

In the first two papers [1,2] the authors tried to employ elliptic rotational coordinates to develop the theory of the method, but they found some disagreements between theory and practice. In the third paper [3] they corrected

\footnotetext{
* Corresponding author. E-mail address: fernando.lameiras@pq.cnpq.br
} 
the theoretical flaws by using the Hankel transform according to Nanis and Kesselman [4]. However, this solution is complex and difficult to explain to most users of this new technique. The authors derived the Legendre's equation by applying the rotational elliptic coordinates to the Laplace's equation, but in the corrected application the Chebyshev's equation is derived. This paper presents the solution of the Chebyshev's equation to this problem, which is derived with simple mathematical methods and agrees with the solution of the Hankel transform.

\section{Theory}

Fig. 1 shows the situation where the face of a disk is immersed in a fluid where there are negative and positive electric charges. Let the face of the disk be made of a kind of material whose surface has affinity for negative electric charges. A layer of negative electric charges adheres to this surface. After that, a layer of positive charges is attracted to this layer of negative charges. However, because of thermodynamic fluctuations, this layer presents disorganization. Another more disorganized layer with negative charges approaches this surface. Successive layers with progressive disorganization will follow, and after a distance $d$, the disorganization between negative and positive charges will be the same as observed in the fluid.

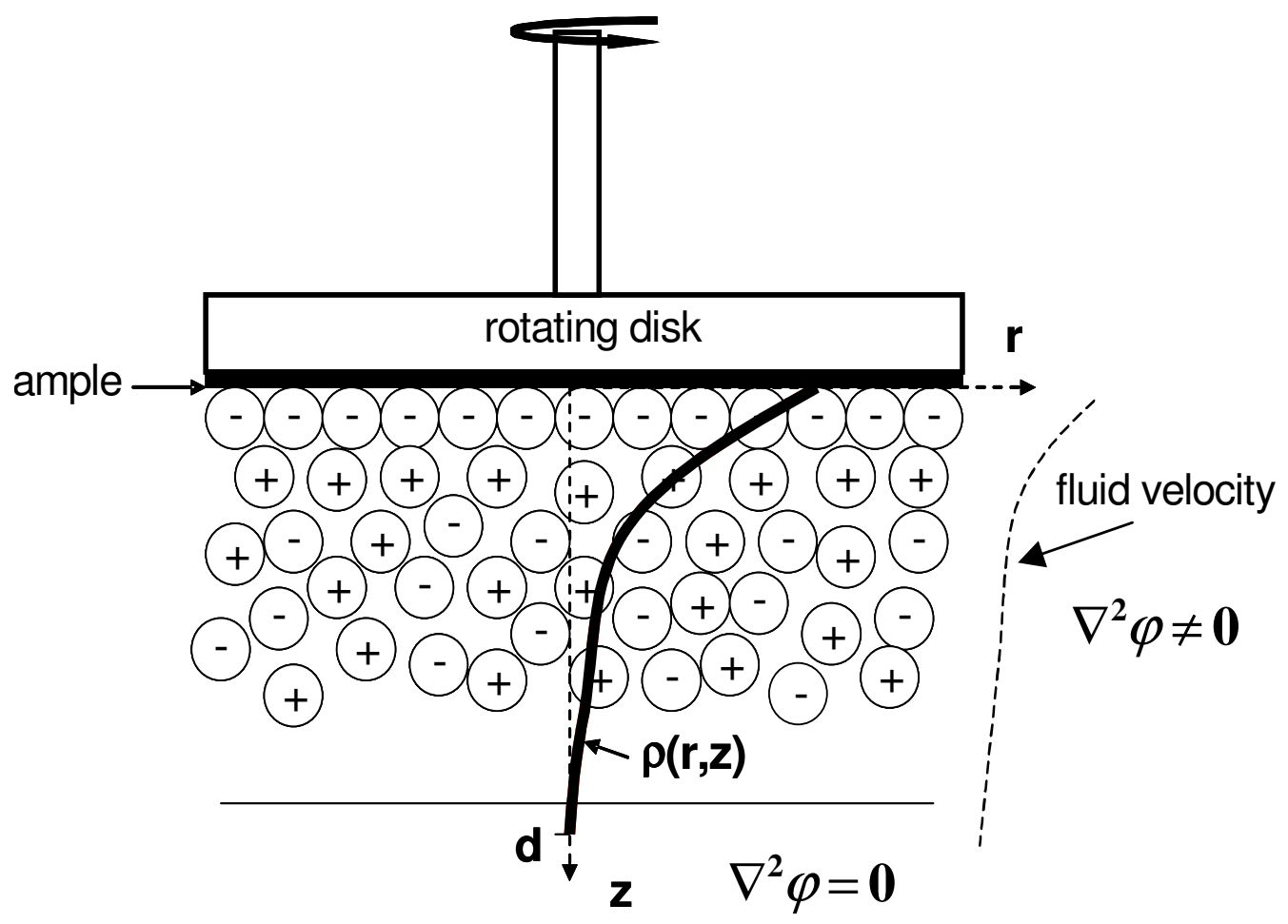

Figure 1. Scheme of the assembly used in the measurement of the zeta potential using a rotating disk. 
The separation between positive and negative charges near the face of the disk generates electric dipoles, whose electric field can be observed. If the disk is rotated, according to the principles of the viscous fluids dynamics, the fluid layer in contact with the disk will rotate at the same velocity as the disk. The layer near the disk will rotate at a lower velocity. The difference between the velocities of these two layers is proportional to the viscosity of the fluid. The velocity of the successive layers decreases in the same way, as shown in Fig. 1.

The rotating movement of the disk generates an outward radial movement of the fluid towards the edge of the disk. The velocity $\mathrm{v}_{\mathrm{r}}$ of this movement is given by $[5,6,7]$

$$
v_{\mathrm{r}}=\gamma r z
$$

$\gamma=0.51023 \Omega^{3 / 2} v^{-1 / 2}$, where $\Omega$ is the angular velocity of the disk, and $v$ is the kinematic viscosity of the fluid. $r$ and $z$ are cylindrical coordinates. This radial movement generates a displacement of electric charges in the vicinity of the disk because, as shown in Fig. 1, there are more free positive electric charges than free negative electric charges. This generates a radial electric current. The charges move outward to the edge of the disk, where they leave this layer and flow back through the liquid toward the disk.

Let $\rho(r, z)$ be the electric charge density in the fluid. This density will be different from zero only in the vicinity of the disk. Therefore, $\rho(r, z)$ will rapidly approach zero when $z$ increases. The radial electric current through a wall of fluid of radius $r$ and height $\Delta z$ is

$$
\Delta i_{\mathrm{r}}=\rho(r, z) v_{\mathrm{r}} 2 \pi r \Delta z
$$

According to Gauss's law, $\rho(r, z)=-\varepsilon \nabla^{2} \varphi$, where $\varphi$ is the electric potential and $\varepsilon$ is the electric permittivity of the fluid. $\nabla^{2} \varphi=(1 / r) \partial / \partial r(r \partial \varphi / \partial r)+\partial^{2} \varphi / \partial z^{2}$ is the Laplacian of the electric potential in cylindrical coordinates with cylindrical symmetry $\left(\partial^{2} \varphi / \partial \theta^{2}=0\right) . \partial \varphi / \partial r$ has a small variation for $r<R(R$ is the radius of the disk). Thus,

$$
\Delta i_{\mathrm{r}} \approx-\varepsilon \frac{\partial^{2} \varphi}{\partial z^{2}} \gamma r z 2 \pi r \Delta z
$$

The radial electric current can be obtained through the integration of (3),

$$
i_{\mathrm{r}}=-\int_{0}^{\infty} 2 \pi r^{2} \varepsilon \gamma \frac{\partial^{2} \varphi}{\partial z^{2}} z \mathrm{~d} z=-2 \pi r^{2} \varepsilon \gamma\left\{\left[z \frac{\partial \varphi}{\partial z}\right]_{0}^{\infty}-[\varphi]_{0}^{\infty}\right\}
$$

This radial current is confined to a layer near the face of the disk. The physical quantity 


$$
\zeta=\left[z \frac{\partial \varphi}{\partial z}\right]_{0}^{\infty}-[\varphi]_{0}^{\infty}
$$

is defined as the zeta potential. The current $i_{\mathrm{R}}$ increases with the square of $r$ until the maximum value $i_{\mathrm{R}}=-2 \pi \varepsilon R^{2} \gamma \zeta$ is reached.

The radial current increment in a ring of the layer of fluid near the surface of the disk is

$$
\Delta i_{\mathrm{r}}=-4 \pi \varepsilon \gamma \zeta r \Delta r
$$

This current increment must be supplied by the fluid below the ring. Thus, the current density perpendicular to the surface of the disk, $j_{z}$, is

$$
j_{\mathrm{z}}=-\frac{\Delta i_{\mathrm{r}}}{2 \pi r \Delta r}=2 \varepsilon \gamma \zeta
$$

The negative sign takes to account that $j_{\mathrm{z}}$ is in the opposite direction of the $\mathrm{z}$ coordinate axis. This equation shows that the current density returning to the layer of fluid near the face of the disk is uniform when $0 \leq r \leq R$.

In a volume element sufficiently distant from the face of the disk, there is a charge balance. Thus, according to Gauss's law, $\nabla^{2} \varphi=0$. This equation can be solved using the elliptical coordinates $\xi$ and $\eta$, where $r=R\left[\left(1+\xi^{2}\right)\left(1-\eta^{2}\right)\right]^{1 / 2}$ and $z=R \xi \eta$. Considering a solution such as $\varphi(\xi, \eta)=M(\xi) P(\eta)$, one obtains Chebyshev's differential equations (with real argument for $\eta$ and imaginary argument for $\xi$ ). Solutions convergent for $\xi \leq 1$ and $\eta \leq 1$ are obtained considering $M(\xi)=\sum_{j=0}^{\infty} a_{j} \xi^{j}$ and $P(\eta)=\sum_{j=0}^{\infty} c_{j} \eta^{j}$, where $j$ is an integer. The general solution is

$$
\varphi(\xi ; \eta ; b)=\left[a_{0} M_{0}(\xi ; b)+a_{1} M_{1}(\xi ; b)\right]\left[c_{0} P_{0}(\eta ; b)+c_{1} P_{1}(\eta ; b)\right]
$$

where

$$
\begin{gathered}
M_{0}(\xi ; b)=\sum_{j=0}^{\infty}\left[b-(2 j-2)^{2}\right]\left[b-(2 j-4)^{2}\right] \cdots[b-0] 1 \frac{\xi^{2 j}}{(2 j) !} \\
M_{l}(\xi ; b)=\sum_{j=0}^{\infty}\left[b-(2 j-1)^{2}\right]\left[b-(2 j-3)^{2}\right] \cdots[b-1] 1 \frac{\xi^{2 j+1}}{(2 j+1) !} \\
P_{0}(\eta ; b)=\sum_{j=0}^{\infty}\left[(2 j-2)^{2}-b\right]\left[(2 j-4)^{2}-b\right] \cdots[0-b] 1 \frac{\eta^{2 j}}{(2 j) !}
\end{gathered}
$$




$$
P_{l}(\eta ; b)=\sum_{j=0}^{\infty}\left[(2 j-1)^{2}-b\right]\left[(2 j-3)^{2}-b\right] \cdots[1-b] 1 \frac{\eta^{2 j+1}}{(2 j+1) !}
$$

The first boundary condition is that the function $\varphi(r ; z)=\varphi(\xi ; \eta ; b)$ must provide a current density perpendicular to the face of the disk equal to $j_{\mathrm{z}}=2 \varepsilon \gamma \zeta$. $j_{\mathrm{z}}=-k\left[\frac{\partial \varphi}{\partial z}\right]_{z=0}=-\frac{k}{R \eta}\left[\frac{\partial \varphi}{\partial \xi}\right]_{\xi=0}$, where $k$ is the electric conductivity of the fluid.

One obtains from (9) that

$$
\left[a_{0} M_{0}^{\prime}(0 ; b)+a_{1} M_{1}^{\prime}(0 ; b)\right] \frac{\left[c_{0} P_{0}(\eta ; b)+c_{1} P_{1 .}(\eta ; b)\right]}{\eta}=-\frac{2 \varepsilon \gamma R}{k}
$$

This equation is true if $\left[c_{0} P_{0}(\eta ; b)+c_{1} P_{1}(\eta ; b)\right] / \eta$ is constant. In order to fulfill this condition, $c_{0}=0$ and $b=1$, because $P_{1}(\eta ; 1)=\eta$. Moreover, $M_{0}^{\prime}(0 ; 1)=0$, $M_{1}^{\prime}(0 ; 1)=1$, and $M_{1}(\xi ; 1)=\xi$. The solution is given by

$$
\varphi(\xi ; \eta ; 1)=a_{0} c_{1} \eta M_{0}(\xi ; 1)-2 \frac{\varepsilon \gamma \zeta R}{k} \eta \xi
$$

The second boundary condition is that the streaming potential is maximum at the center of the disk $(r=0 ; z=0)$ or $(\eta=1 ; \xi=0)$ and is equal to $\frac{\varepsilon \gamma \zeta R}{k}$, so that

$$
\varphi(\xi ; \eta ; 1)=\frac{\varepsilon \gamma \zeta R}{k} \eta\left[M_{0}(\xi ; 1)-2 \xi\right]
$$

\section{Discussion}

Equation (12) is true where $\nabla^{2} \varphi=0$, that is, where there is electric charge balance in a volume of fluid. This situation exists for $z>d$ from the face of the disk (Fig. 1 ). In the region between $z=0$ and $z=d, \nabla^{2} \varphi \neq 0$, that is, there is a net electric charge. The radial electric current is confined to this region. $d$ is related to the Debye's distance in an electrolyte [8]. In Fig. 1, $d$ is shown as amplified for didactical purposes, because $d<<<R$. The electric potential $\varphi(\xi ; 1 ; 1)$ can be measured by putting an electrode in $(z, r)=(R \xi ; 0)$ and another one in a position $z>>R$, where $\varphi(z, r)=0$.

Along the axis of the disk $(\eta=1)$, equation (12) gives that

$$
\frac{\varphi(\xi ; 1 ; 1) k}{\varepsilon \gamma \zeta R}=M_{0}(\xi ; 1)-2 \xi
$$

The right hand side of (13) gives the same values for small $\xi$ as the approximated function employed by Sides, 


$$
\frac{\varphi(\xi ; 1 ; 1) k}{\varepsilon \gamma \zeta R} \approx 2\left(1-\xi-\frac{1}{2 \sqrt{1+\xi^{2}}}\right)
$$

$\varphi(\xi ; 1 ; 1)$ is typically around $0.1 \mathrm{mV}$ near the disk and falls as $\xi$ growths. This streaming potential is not very high relative to the noise observed in practice. So, $\xi$ should be as close as possible to zero in order to keep the streaming potential as high as possible to decrease the error of measurement. Another good procedure is to give an identifiable pattern to the rotation of the disk (e. g., a sinusoidal pattern) to separate the streaming potential from the noise.

\section{Conclusion}

This paper corrects the approaches previously published by using elliptic rotational coordinates to calculate the streaming potential near a rotating disk immersed in a fluid with electric charges.

The rotation of a disk whose radius is $R$, immersed in a fluid where there are negative and positive electric charges, generates an electric potential difference between a position $z$ along the axis of the disk and another position distant from the disk given by (for $z \leq R$ )

$$
\Delta \varphi\left(\frac{z}{R}\right)=\frac{\varepsilon \gamma \zeta R}{k}\left[M_{0}(\xi ; 1)-2\left(\frac{z}{R}\right)\right]
$$

where $\Delta \varphi(z / R)$ is the electric potential difference, $\varepsilon$ is the electric permittivity of the fluid, $k$ is the electric conductivity of the fluid, $\zeta$ is the zeta potential of the surface of the disk, $R$ is the radius of the disk, $\gamma=0.51023 \Omega^{3 / 2} v^{-1 / 2}$, where $\Omega$ is the angular velocity of the disk and $v$ is the kinematic viscosity of the fluid, and

$$
M_{O}\left(\frac{z}{R}\right)=\sum_{j=0}^{\infty}\left[1-(2 j-2)^{2}\right]\left[1-(2 j-4)^{2}\right] \cdots 1 \cdot 1 \frac{1}{(2 j) !}\left(\frac{z}{R}\right)^{2 j}
$$

$\Delta \varphi, v$ and $k$ can be measured, whereas $\Omega, R$ and $z$ can be controlled. In these conditions, it is possible to calculate the zeta potential with equation (15) by measuring the electric potential in a position along the axis of the disk.

\section{Acknowledgments}

To CNPq (Processes n. 472027/2004-2 and 305811/2006-1) and to CAPES.

\section{References}

1. P.J. Sides and J.D. Hoggard, Langmuir 20 (2004) 11493-114938.

2. J.D. Hoggard, P.J. Sides and D.C. Prieve, Langmuir 21 (2005) 7433-7438.

3. P.J. Sides, J. Newman, J.D. Hoggard and D.C. Prieve, Langmuir 22 (2006) 9765-9769. 
4. $\quad$ L. Nanis and W. Kesselman, J. Electrochem. Soc. 118 (1971) 454-461.

5. Th. Von Kármán, Z. Angew. Math. Mech. 1 (1921) 233-252.

6. W.G. Cochran, Proc. Cambridge Philos. Soc. 20 (1934) 365-375.

7. $\quad$ M.H. Rogers and G.N. Lance, J. Fluid Mech. 7 (1960) 617-631.

8. R.J. Golston and P.H. Rutherford, Introduction to Plasma Physics, Institute of Physics Publishing, Philadelphia, 1977. 